\title{
Size-Dependence of the Melting Temperature of Individual Au Nanoparticles
}

Schlexer, Philomena; Andersen, Anton Bay; Sebok, Bela; Chorkendorff, Ib; Schiøtz, Jakob; Hansen, Thomas W.

Published in:

Particle \& Particle Systems Characterization

Link to article, DOI:

$10.1002 /$ ppsc.201800480

Publication date:

2019

Document Version

Peer reviewed version

Link back to DTU Orbit

Citation (APA):

Schlexer, P., Andersen, A. B., Sebok, B., Chorkendorff, I., Schiøtz, J., \& Hansen, T. W. (2019). SizeDependence of the Melting Temperature of Individual Au Nanoparticles. Particle \& Particle Systems Characterization, 36(3), [1800480]. https://doi.org/10.1002/ppsc.201800480

\section{General rights}

Copyright and moral rights for the publications made accessible in the public portal are retained by the authors and/or other copyright owners and it is a condition of accessing publications that users recognise and abide by the legal requirements associated with these rights.

- Users may download and print one copy of any publication from the public portal for the purpose of private study or research.

- You may not further distribute the material or use it for any profit-making activity or commercial gain

- You may freely distribute the URL identifying the publication in the public portal 


\section{WILEY-VCH}

DOI: 10.1002/ ((please add manuscript number))

Article type: Full Paper

\section{Size-dependence of the melting temperature of individual Au nanoparticles}

Philomena Schlexer, Anton Bay Andersen, Bela Sebok, Prof. Ib Chorkendorff, Prof. Jakob Schiøtz and Thomas W. Hansen*

P. Schlexer, Bela Sebok, I. Chorkendorff, J. Schiøtz

Department of Physics

Technical University of Denmark

Kgs. Lyngby, DK-2800, Denmark
A. B. Andersen, T. W. Hansen
Center for Electron Nanoscopy
Technical University of Denmark
Kgs. Lyngby, DK-2800, Denmark

Keywords: melting, gold, nanoparticles, transmission electron microscopy, molecular dynamics simulations

Nanoparticles have an immense importance in various fields, such as medicine, catalysis and various technological applications. Nanoparticles exhibit a significant depression in melting point as their size goes below ca. $10 \mathrm{~nm}$. However, nanoparticles are frequently used in high temperature applications such as catalysis where temperatures often exceed several 100 degrees which makes in interesting to study not only the melting temperature depression, but also how the melting progresses through the particle. Using high resolution transmission electron microscopy, we have investigated the melting process of gold nanoparticles in the size range 2-20 nm Au nanoparticles combined with molecular dynamics studies. We confirm a linear dependence of the melting temperature on the inverse particle size and electron microscopy imaging reveals that the particles start melting at the surface and the liquid shell formed then rapidly expands to the particle core.

\section{Introduction}




\section{WILEY-VCH}

A fundamental understanding of the thermodynamic properties of nanoparticles is of special interest because of their importance in a broad range of applications, from industrial catalysis to medicine [1]. Nanomaterials exhibit properties differing from their bulk counterparts due to their reduced size. An interesting property, which can be largely affected by the material dimensions, is the melting temperature. Based on both experimental and theoretical studies, it has been well established that the melting temperature of nanoparticles depends on their size [2]. In particular, metal nanostructures typically exhibit a melting temperature depression with respect to their bulk counterparts [3]. Of additional interest is the actual melting mechanism, i.e. how the melting proceeds through the particle and can a molten skin be maintained on top of a solid core.

Theoretical [4-9] computational [10-14] and experimental [15, 16] studies have been performed to elucidate the melting mechanism as well as the exact dependency of the melting temperature on the particle diameter. The reported melting temperature of free-standing metal nanoparticles is almost always linearly dependent on the inverse particle diameter $\left(\mathrm{T}_{\mathrm{m}} \sim \mathrm{d}^{-1}\right)$, see e.g. the review by Nanda [2] or the perspective by Li et al. [7] Furthermore, Hendy suggested that the melting temperature of supported metal particles is the same as those of free spherical particles with the same surface curvature [17].

Several thermodynamic models were developed to describe the melting temperature of nanoparticles, such as the Pawlow model [18], the Rie model [19] and the model proposed by Reiss and Curzon [20]. [J. Nanopart. Res. 2017, 19: 263, J. Phys. Chem. C 2018, 122, 15713-15722; J. Colloid Interf. Sci. 2001, 243, 388-390; J. Phys. Chem. C 2011, 115, 2279622803] However these models typically fail to predict the melting temperature of smaller nanoparticles ( $d<5 \mathrm{~nm}$ ), due to the introduction of approximations in their derivation [4].

This study applies a combined experimental and computational approach to investigate the melting properties of Au nanoparticles as a function of particle size. Experimental approaches reported in the literature are typically based on averaging methods to determine the melting 


\section{WILEY-VCH}

point [4, 21]. For example, Buffat and Borel [21] measure the disappearance of diffraction rings from an ensemble of nanoparticles, and later determine the size distribution with electron microscopy. Here we instead measure the melting point of individual nanoparticles with known size using atomistic investigation methods. To establish a precise relation between melting temperature and particle size, gas-phase mass-selected nanoparticles [22] were soft-landed on a heatable Micro-Electro-Mechanical System (MEMS) [22]. The experiments are complemented with Molecular Dynamics (MD) simulations, investigating the melting of nanoparticles of different shapes and modeled with two different interatomic potentials. The melting temperatures of 2-20 nm Au nanoparticles are computed and the melting mechanism is characterized. Finally, the experimental and theoretical results are compared to a thermodynamic model suited for the melting mechanism found.

\section{Results and Discussion}

Mass-selected gold nanoclusters were produced at the cluster source at DTU [23], described in the Section 4. They were produced to have an average particle diameter of $1.9 \mathrm{~nm}, 3.0 \mathrm{~nm}$ and $5.0 \mathrm{~nm}$ respectively. The advantage of using mass-selected clusters is that the statistical spread on the measured melting point will be very small and the same nanoparticle does not need to be followed at all temperatures. We measured the diameter of each individual particle of which we determined the melting temperature. In order to minimize handling, clusters were deposited directly onto a SiN substrate in the MEMS heating devices and inserted in a DENSsolutions Wildfire heating holder. The compact design of such a device minimizes thermal drift and allows for high spatial resolution imaging, even when changing the temperature.

The samples were imaged in an FEI Titan E-Cell 80-300 ST transmission electron microscope equipped with an image corrector giving a resolution better than $1 \AA$. For image acquisition, the microscope is equipped with a Gatan OneView camera, which allows one to 


\section{WILEY-VCH}

follow the particles in real time and correlate each frame to a temperature log to get the temperature at which a specific event occurred. A frame rate of 5 fps was used, which allows a good time resolution while still having sufficient contrast to see lattice fringes. The microscope was operated at $300 \mathrm{kV}$ and image sequences were acquired at an electron flux density of approx. $4 \times 10^{5} \mathrm{e}^{-/ \mathrm{nm}^{2} \mathrm{~s}}$.

The experiments consisted of several temperature cycles. In each cycle, the temperature was first increased to $863 \mathrm{~K}$ over 2 minutes. The temperature was then gradually increased to 888K in small increments in order to minimize the sample drift allowing for image acquisition with atomic resolution. When the nanoparticles were molten and the exact temperature logged, the sample was cooled to $573 \mathrm{~K}$ over 2 minutes and allowed to equilibrate for approximately 15 minutes. The cycle was then repeated.

The state of the nanoparticles was determined by the presence or lack of crystalline lattice fringes in the micrographs. Whereas this may seem a crude method, it provides data of a local nature. By analyzing several particles of the same size, a lower limit of the melting point can be determined. Melting temperatures were determined as the lowest temperature where lattice fringes were no longer visible. At low temperatures, the lattice fringes were continuously observable and most particles had the dodecahedral shape typical of gold nanoparticles [24]. From roughly $100 \mathrm{~K}$ below the melting point, regions with lattice fringes shifted within the nanoparticle, but were almost continuously observed and most particles had an ill-defined surface layer not observed at low temperature. Above the melting point, lattice fringes were no longer observed. After the frame where lattice fringes were no longer observed, each nanoparticle in the dataset was observed for a period of about 1 minute to ensure that the particle was indeed molten.

Whereas all nanoparticles are observed to be completely crystalline throughout their extent at room temperature, Figure $\mathbf{1}$ a and b, they develop amorphous surface regions approximately $100 \mathrm{~K}$ below the melting point. These are seen in Figure 1c and $\mathrm{d}$ as regions 


\section{WILEY-VCH}

without lattice fringes. As the temperature is increased and approaches the melting point, the thickness of the molten layer increases slightly.

As the temperature reaches the melting point, the lattice fringes vanish and do not reappear, see Figure 2. This typically happens from frame to frame within the image sequence, i.e. in less than $0.2 \mathrm{~s}$.

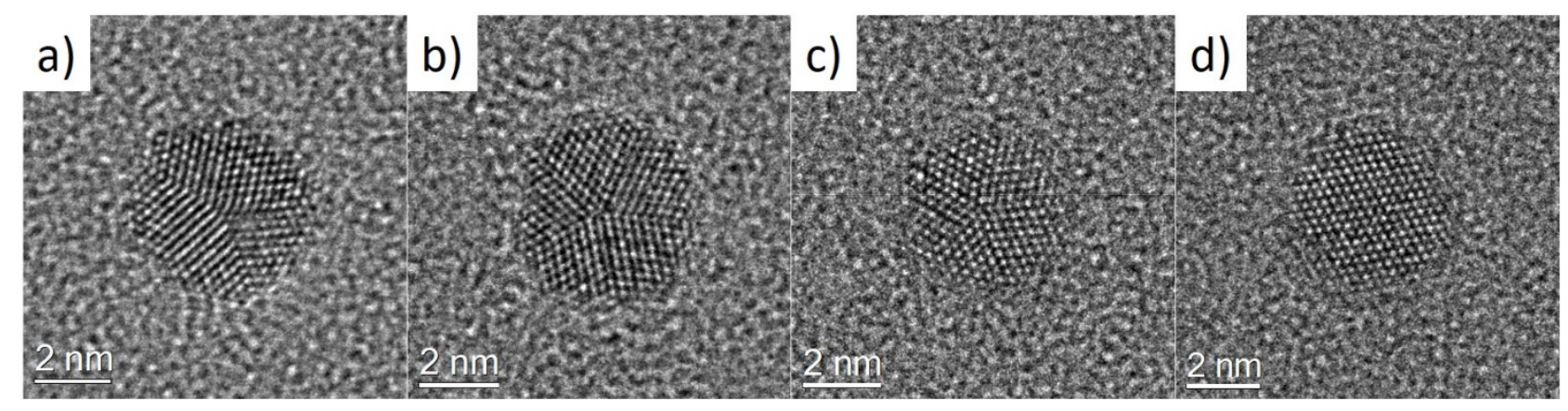

Figure 1: Gold nanoparticles imaged at a) $296 \mathrm{~K}$, b) $773 \mathrm{~K}$, c) $873 \mathrm{~K}$ and d) $885 \mathrm{~K}$ respectively. An amorphous surface region is observed from $773 \mathrm{~K}$ and above. Most frames show the five-fold symmetry with (111)-type planes typical of gold nanoparticles exposed.

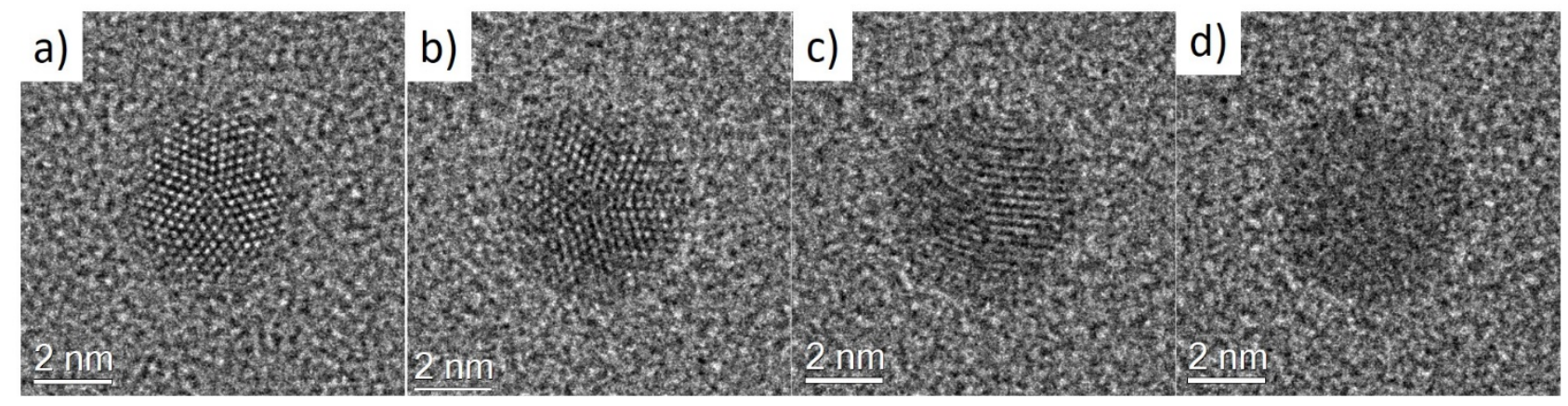

Figure 2: Frames from a single image sequence showing the melting of a gold nanoparticle Images are acquired at $885 \mathrm{~K}$. Initially, (111) lattice fringes are clearly visible along with the amorphous surface, a) and b). These fringes disappear from c) to d) separated by $0.2 \mathrm{~s}$.

Figure 3 shows two nanoparticles of the desired size, and one with twice the volume. The latter is formed when two particles coalesce into one or during particle formation in the gas aggregation source (see Supporting Information). The upper row shows the images of the three particles, recorded at $888 \mathrm{~K}$. The middle row is the Fast Fourier Transform (FFT) of the 


\section{WILEY-VCH}

upper right particle, in (d) the lattice spots show a clear decahedral symmetry, consistent with a particle of decahedral shape, as is often seen for medium-sized nanoparticles [25]. In (e), the Bragg peaks are almost gone, and in (f) only rings are seen, consistent with an amorphous or liquid structure. The lower row shows the FFT of the lower left particle with twice the volume. It is slightly off-axis, so lattice fringes are only seen in one direction resulting in two spots in the FFT. These spots remain stable at $888 \mathrm{~K}$, indicating that the melting temperature of this larger particle is higher.
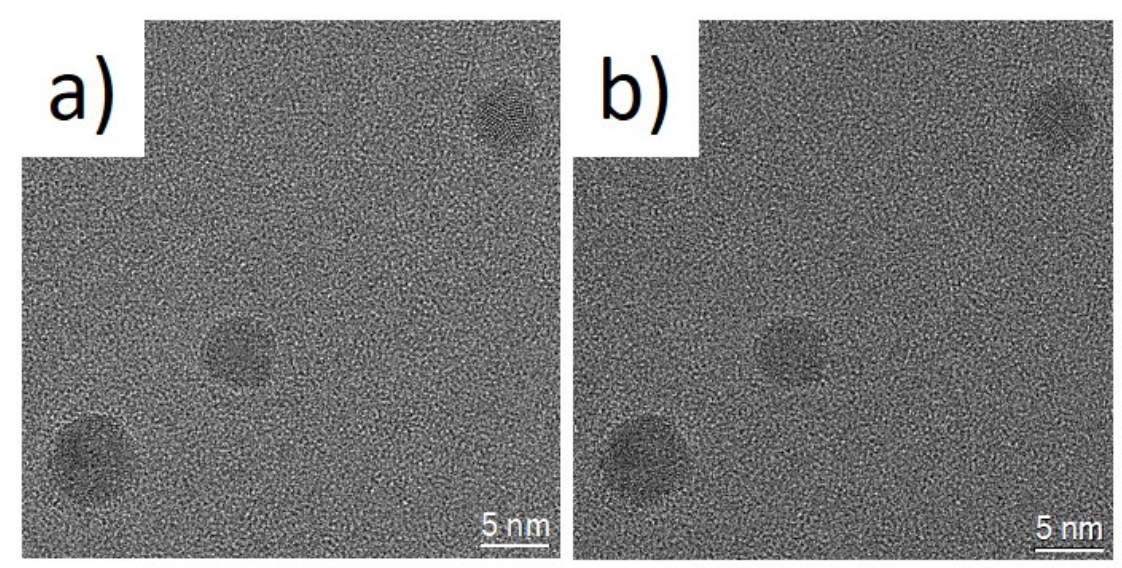

c)
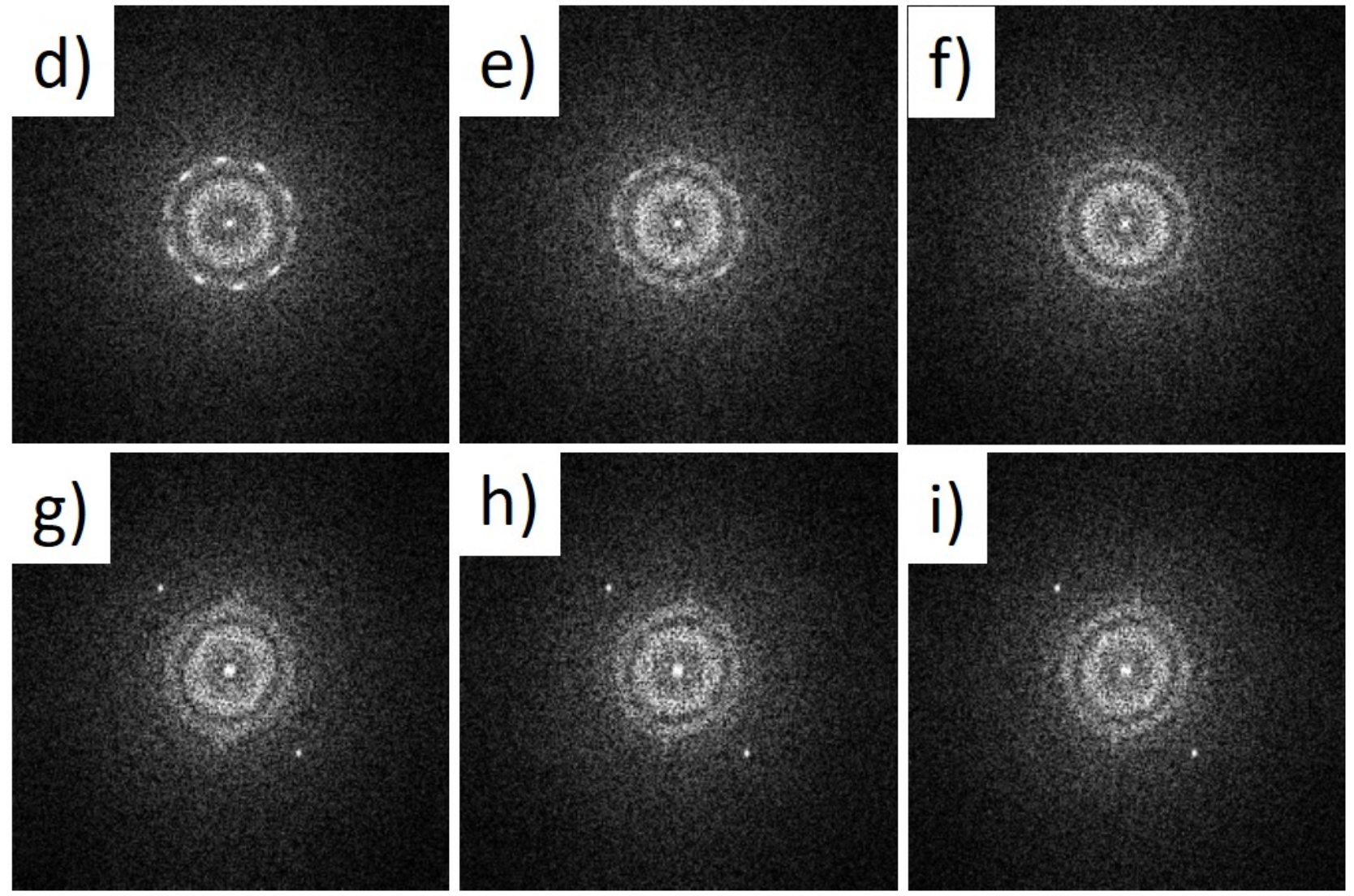


\section{WILEY-VCH}

Figure 3: Sequence of images recorded at $888 \mathrm{~K}$, a)-c) along with FFTs of the upper right particle, d)-f) and lower left particle g)-h). The $5 \mathrm{~nm}$ particle in the upper right part of the image shows several sets of (111) fringes up until frame e). After this frame, no fringes are observed for this particle. The larger particle in the lower left of the image, exposes (220) lattice fringes throughout the sequence.

To investigate the effect of the electron beam on the measured melting temperature, we determined the melting temperature of particles which have similar size, at different electron beam intensities (between $2 \cdot 10^{5}$ and $8 \cdot 10^{5} \mathrm{e}^{-} / \mathrm{nm}^{2}$ ). The results are summarized in Figure 6 . No substantial effect of the beam was observed for particles with $d \leq 2 \mathrm{~nm}$, i.e. the melting temperature did not change when increasing the electron beam intensity, as shown in Figure S1.

The particle shape has a significant influence on the melting temperature, as it affects the surface energy. Roling et al. have shown how the binding energy of surface atoms of the nanoparticle depends on their coordination number [26]. The ratio of atoms in lowercoordinated sites (edges and vertexes) increases with inverse particle diameter and this results in a gradual decrease in surface energy [27]. This in turn directly affects the melting temperature. Although a few thermodynamic models have been proposed, no consensus on the exact functional of the melting temperature on the particle geometry and chemical compositions has been established so far. That is, the exact functional form remains elusive.

The TEM experiments were complemented with molecular dynamics simulations of the melting of gold nanoparticles with different sizes, as described in the Section 4. Two different interatomic potentials for gold were used, the effective medium theory (EMT) [28] and the embedded atom method (EAM) [29]. Both potentials belong to the same family of manybody potentials, and have long been used to describe metallic systems. As the potentials are 


\section{WILEY-VCH}

fitted to low-temperature properties such as elastic constants, binding energies and surface energies, they cannot be expected to reproduce the melting temperature perfectly but will display the correct trends. In the experiments, the particle shapes can deviate from a perfect Wulff-construction, for instance because the number of atoms does not match the number needed for a perfect Wulff-construction. To account for at least two different nanoparticle shapes in the molecular dynamics simulations, we also investigated round particles.

In Figure 4, the melting process of a $2.97 \mathrm{~nm}$ gold nanoparticle containing 807 atoms in a Wulff-constructed shape is shown. The particle is heated gradually, resulting in the increase of the instantaneous temperature (calculated from the average kinetic energy) and the cohesive energy of the particle, respectively, as shown in Figure 4a. Before and after the melting region, the cohesive energy increases linearly with the temperature. The slope of this linear increase is the heat capacity $\left(\mathrm{C}_{\mathrm{p}}\right)$ and has a value of approx. $\mathrm{C}_{\mathrm{p}}=2.7 \times 10^{-4} \frac{\mathrm{eV}}{\mathrm{K}}$, which is consistent with what is expected for a solid above the Debye temperature $\left(3 \mathrm{k}_{\mathrm{B}}=2.6 \times 10^{-4} \frac{\mathrm{eV}}{\mathrm{K}}\right)$. In the melting region, the instantaneous temperature of the particle decreases slightly, see points $\mathbf{P}_{\mathbf{1}}$ to $\mathbf{P}_{\mathbf{3}}$ in Figure 4 a. The fact that the temperature decreases between $\mathbf{P}_{\mathbf{1}}$ and $\mathbf{P}_{\mathbf{3}}$, giving a negative heat capacity, is due to finite size effects, in particular the curvature of the solid-liquid interface $[14,30]$. We fitted the melting curve (blue continuous line) with a second order fit function (black dashed line) around the melting region, as shown in Figure 4a, and we defined the maximum of this fit function $\left(\mathbf{P}_{\mathbf{1}}\right)$ as the melting temperature, as the particles are already completely molten at $\mathbf{P}_{\mathbf{3}}$. With this method, we have a mathematical and systematically applicable way to determine the melting temperature. For this $3.0 \mathrm{~nm}$ gold particle, we obtain a melting temperature of $763 \mathrm{~K}$. The procedure to determine the melting temperature was also applied to the other particle sizes and shapes. In all cases, we define the particle diameter from the number of atoms via $\mathrm{d}=$ 


\section{WILEY-VCH}

$2 \cdot \mathrm{rws} \cdot \mathrm{N}^{1 / 3}$, where $\mathbf{r}_{\mathbf{w s}}$ is the Wiegner-Seitz radius of bulk gold, and $\mathrm{N}$ is the number of atoms.

This way, different particles shapes are directly comparable.
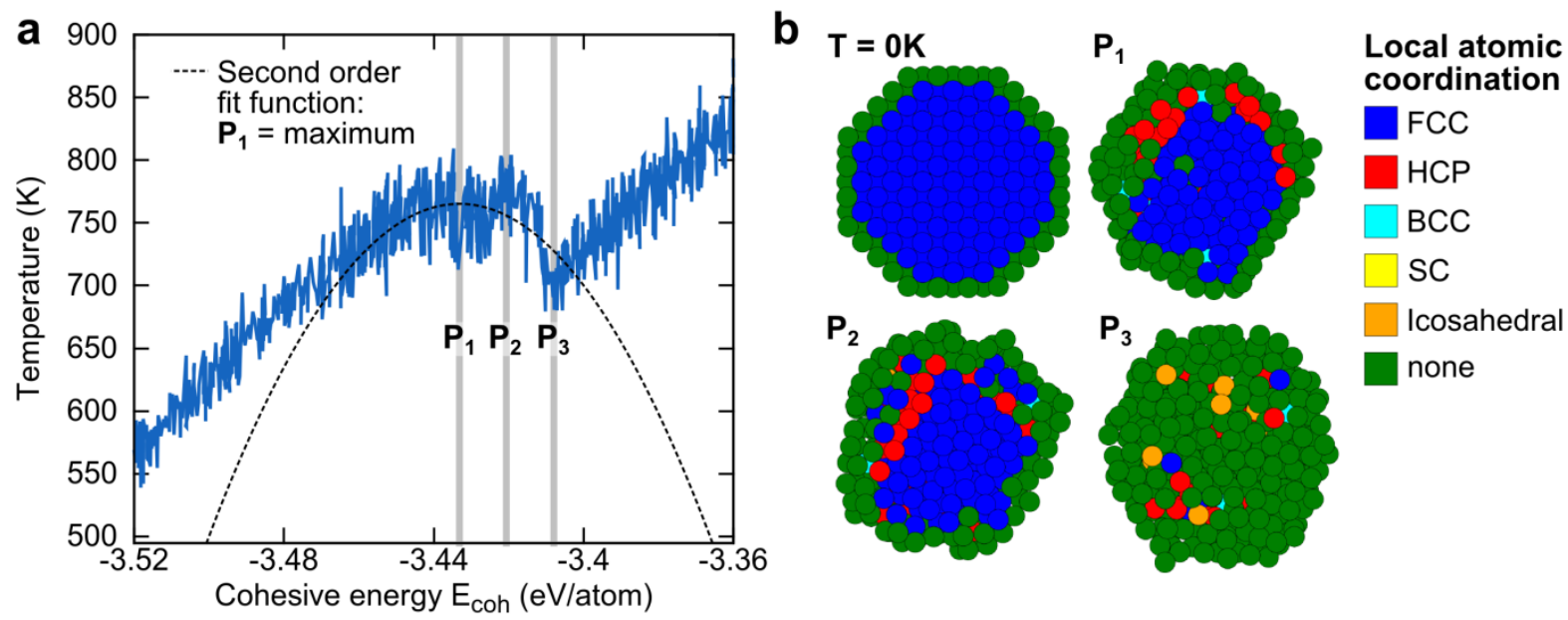

Figure 4: Detailed analysis of the melting process of a Wulff-constructed Au nanoparticle ( $d=$ $3.0 \mathrm{~nm}, \mathrm{~N}=807$ atoms), simulated with an interatomic potential based on the effective medium theory (EMT) [28]. a The instantaneous temperature is plotted against the cohesive energy of the nanoparticle (continuous blue curve). In the melting region, the curve is fitted via a second order fit function (dotted black curve). b Polyhedral template matching (PTM) was used to determine the local atomic coordination at $\mathrm{T}=0 \mathrm{~K}$ and at three different points in the melting region ( $\mathbf{P}_{\mathbf{1}}$ to $\mathbf{P}_{3}$ ), which are specified by the grey bars in $\mathbf{a}$.

To understand the melting mechanism in more detail, we investigated the arrangement of the atoms in the nanoparticle during the melting process. This can be done with polyhedral template matching (PTM), which is a powerful tool to analyze the atomic coordination environment of the atoms also at elevated temperatures [31]. In Figure 4b, cross-sections of a Wulff-shaped Au nanoparticle at different stages during the melting process are shown. At T $=0 \mathrm{~K}$, the nanoparticle exhibits a perfect Wulff-construction shape and all atoms show a perfect FCC crystalline atomic environment, except the surface atoms which are missing some neighbors. At $\mathbf{P}_{\mathbf{1}}$, the sub-surface atoms of the nanoparticle lose their perfect FCC 


\section{WILEY-VCH}

crystalline atomic environment and we can observe stacking faults and parts with a complete loss of crystallinity. This indicates that the particle starts melting at the surface under formation of a liquid shell, which is observed in the TEM images as well (Figure 1). At $\mathbf{P}_{2}$, the surface melting proceeds further towards the core of the particle showing the growth of the liquid shell. At $\mathbf{P}_{3}$, the particle is completely molten with a complete loss of crystallinity in the atomic arrangement of the nanoparticle. The findings agree well with molecular dynamics simulations of $\mathrm{Cu}$ and Ag nanoparticles reported in the literature [10, 14].

We determined the melting temperature of gold nanoparticles of different sizes experimentally and computationally. In the latter case, the melting temperatures were determined for both Wulff-constructed and round shaped Au nanoparticles with diameters in the range of 2-20 nm, using the two different interatomic potentials. The resulting melting temperatures are summarized in Figure 5a.
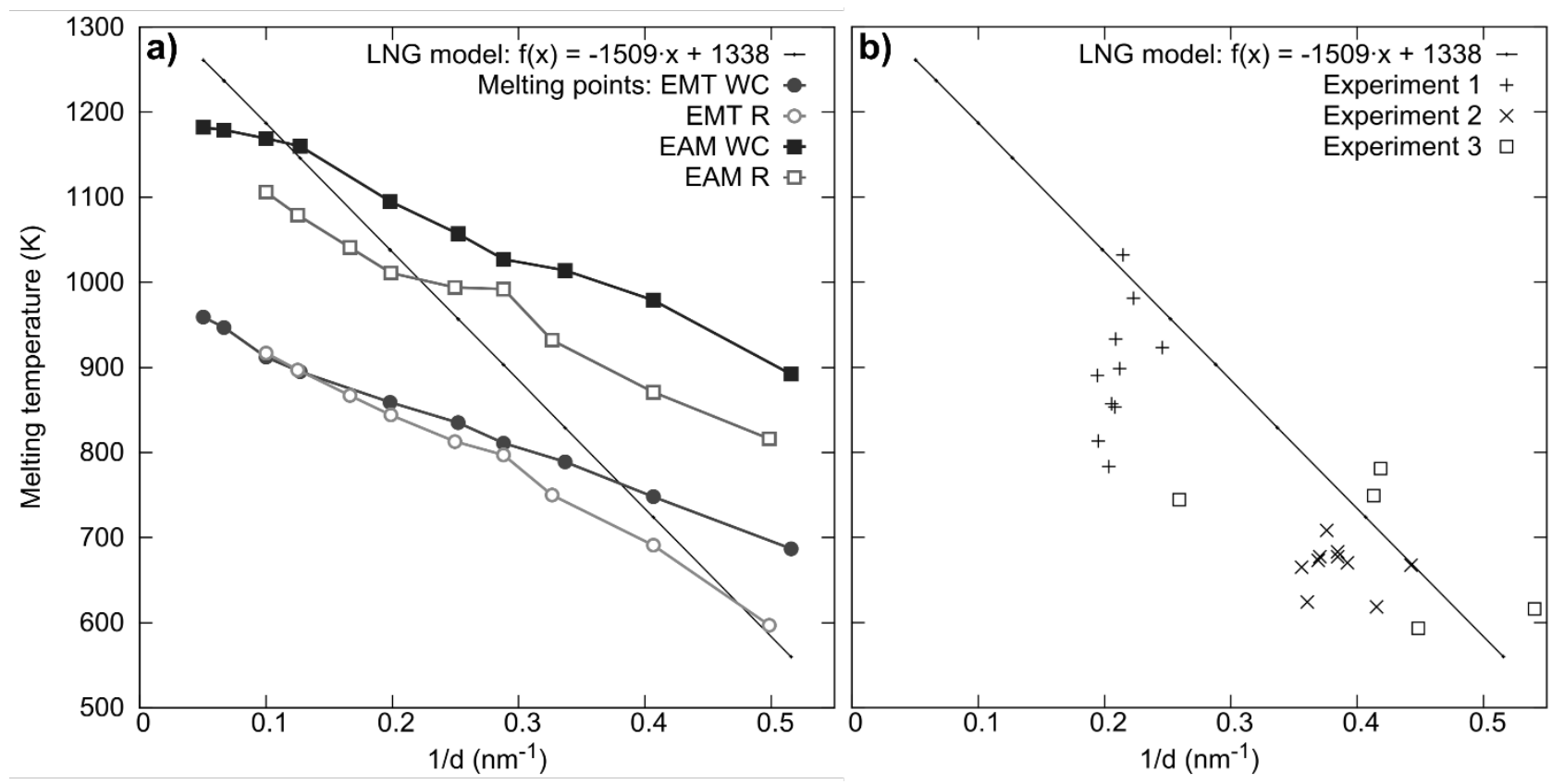

Figure 5: a) Simulated melting temperatures of Au nanoparticles in dependence of the inverse particle diameter (1/d) for each shape (Wulff-construction $=$ WC and round $=\mathrm{R}$ ) and each interatomic potential. Melting temperatures predicted by the liquid nucleation and growth 


\section{WILEY-VCH}

(LNG) model with parameters taken from Nanda [2], which reproduces the experimental bulk melting temperature of $1338 \mathrm{~K}$ [32] at $\mathrm{d} \rightarrow 0$. b) Experimentally determined melting points of Au nanoparticles, together with the LNG model.

In all cases, we observe an approximately linear dependence of the melting temperature on the inverse particle diameter $1 / \mathrm{d}$. The melting temperatures agree well with those reported in the literature [33, 34]. We find that the EAM potential gives melting temperatures roughly $200 \mathrm{~K}$ higher than the EMT potential. The difference in melting temperature of the two potentials is, may be related to the fact that the EMT potential shows a larger underestimation of the surface energy with respect to experiments than the EAM potential, compare refs. [28] and [29]. We furthermore observe that the Wulff-constructed particles exhibit significantly higher melting temperatures than the round particles, although this difference is smaller than the difference caused by the potentials. The differences in the melting temperature can be explained by the difference in the cohesive energy of the nanoparticles, which is smaller for round shapes than for Wulff-constructions with the same number of atoms. Empirically, a correlation of the melting temperature $\left(\mathrm{T}_{\mathrm{m}}\right)$ and the cohesive energy $\left(\mathrm{E}_{\mathrm{coh}}\right)$ of bulk materials according to $T_{m}=\left(0.032 / \mathrm{k}_{\mathrm{B}}\right) \cdot \mathrm{E}_{\mathrm{coh}}$ was found [35], i.e. a smaller cohesive energy results in a lower melting temperature.

In the literature, different thermodynemic models for the melting mechanisms of nanoparticles were proposed [2]. Amongst these are the homogeneous melting model, the liquid skin melting model, and the liquid nucleation and growth model. According to the homogeneous melting model, the particle melts homogeneously, i.e. at the same time at the surface and in the interior of the nanoparticle. The liquid skin melting model states that a liquid skin is formed at the particle surface, followed by a homogeneous melting of the rest of the particle. The liquid nucleation and growth (LNG) predicts the formation of a liquid skin which expands towards the center of the particle until the particle is completely molten. The 


\section{WILEY-VCH}

atomic coordination analysis shown in Figure 4b clearly indicates the formation and growth of a liquid skin, which corresponds to the LNG model. A quantitative representation of the model is given in eqn. (1) [2].

$$
\frac{T_{m}(\mathrm{NP})}{T_{m}(\mathrm{bulk})}=1-\frac{6 V}{\Delta H_{f} d}\left[\gamma_{S v}-\gamma_{l v}\left(\frac{\rho_{S}}{\rho_{l}}\right)^{1 / 2}\right]=1-\frac{\beta_{L N G}}{d}
$$

(Error!

Bookmark not

defined.Error! Bookmark not defined.1)

Here, $\mathrm{V}$ is the volume of the particle, $\Delta \mathrm{H}_{\mathrm{f}}$ is the bulk heat of fusion, $\gamma_{\mathrm{sv}}$ and $\gamma_{\mathrm{lv}}$ are surface energies of solid-vapor and liquid-vapor interfaces, respectively, $\rho_{\mathrm{sv}}$ and $\rho_{\mathrm{lv}}$ are the densities of solid and liquid, respectively. $\mathrm{d}$ is the particle diameter. We can now compare the prediction of the LNG with our results. For this, eqn. (1) is also plotted in Figure 5 a) and b). We used the parameters $\beta_{\text {LNG }}$ and the bulk melting temperatures $T_{m}$ (bulk) from Nanda et al.

[36] The LNG model predicts a steeper slope than the simulations, but the range of temperatures fit the simulated values. This is in agreement with MD simulations performed by Lewis et al. [34].

The melting temperatures of the gold nanoparticles based on the TEM images are shown in Figure 5b. We find a good agreement between the experimentally determined melting temperatures and those obtained via the molecular dynamics simulations. Despite significant scatter due mainly to the analysis technique, the experimentally determined melting temperatures roughly follow a linear dependence on the inverse particle diameter and are in the region predicted by the LNG model. The spread of the experimental data and the fact that most of the data point lay below the LNG line may be related to the fact that the melting temperature is defined as the lowest temperature at which lattice fringes are absent. Due to the dynamical behavior of the particles, this should result in a lower limit for the true melting temperature. Duan et al. [37] found a linear dependence of the melting temperature on the inverse particle diameter for round nanoparticles with a diameter larger than $10 \mathrm{~nm}$. For 


\section{WILEY-VCH}

smaller particles, they found a more severe decrease in the melting temperature, which might be related to sintering as the heat produced via sintering may lead to an underestimation of the effective heat needed to melt the particles.

\section{Conclusion}

In conclusion, the dependence of the melting temperatures of gold nanoparticles on the particle diameter was investigated via molecular dynamics simulations and high-resolution transmission electron microscopy imaging while heating the mass-selected gold nanoparticles. In the computations, two different particle shapes (Wulff-constructions and round particles) were investigated in the size range of 2-20 $\mathrm{nm}$. The melting of each particle was performed with the EMT and the EAM interatomic potential, respectively. We find that the choice of the potential has a larger effect on the simulated melting temperature than the particle shape, although the latter also significantly alters the melting temperature. The experimental melting temperatures lay between the melting temperatures predicted by the EAM and those predicted by the EMT potential. Thus, despite the significant scatter in the experimental results, all in all we observe a good agreement between computations and experiments. In all cases we find a linear dependence of the melting temperature on the inverse particle diameter. With the help of polyhedral template matching and atomistic TEM imaging, we furthermore establish that the melting proceeds from the surface to the center of the particles, corresponding the liquid nucleation and growth model.

\section{Experimental Section}

Nanoparticle synthesis and characterization

Pt and Au nanoparticles were mass selected and deposited using a gas aggregation magnetron sputtering cluster source equipped with a lateral time of flight mass filter (Nano-Beam 2011, Birmingham Instruments Ltd., United Kingdom) [22, 38, 39] which has a base pressure in the 


\section{WILEY-VCH}

low $10^{-10}$ mbar range and a mass resolution of $\mathrm{m} / \Delta \mathrm{m} \geq 20$. The metallic gold target is sputtered by argon plasma and the sputtered species aggregate into nanoparticles of different sizes in a liquid nitrogen cooled aggregation zone [38]. To facilitate the heat transfer between the chamber walls and the metal particles in the aggregation zone, helium gas is supplied. After undergoing supersonic expansion, the particles are focused into the mass filter, which separates the particles based on their mass to charge ratio [39]. Vast majority of the negative particles carry a single charge (an extra $\mathrm{e}^{-}$), but some particles carry double this charge. If the double charged particles have double the mass as well, they pass through the filter together with the single mass-single charge particles, as their mass to charge ratio is the same, causing a small number of double mass particles to appear on the sample surface (Figure 3). The mass filter is connected to an OMICRON Multiscan Lab XP system with a base pressure in the low $10^{-11}$ mbar range for deposition. The particles were deposited on Wildfire Nano-Chip XT MEMS SiNx TEM windows (DENSsolutions, Netherlands) with a kinetic energy of $45 \mathrm{eV}$. These nanochips provide fast and accurate (within a few K) temperature control between RT and around $1600 \mathrm{~K}$. The samples were investigated in a FEI Titan E-Cell 80-300 ST TEM equipped with a post objective lens spherical aberration corrector. The masses of the nanoparticles were chosen to fit the Wulff-constructed Au particles, as predicted by the molecular dynamics simulations. We made sure that no reaction of the Au particles with the substrate occurred by comparing the inter-planar distances of the nanoparticles with those of bulk gold which showed that the nanoparticles are pure gold particles also at higher temperatures. In Figure 6, the effect of varying beam intensities on the melting temperature of the nanoparticles is shown. The expected correlation between electron beam intensity and melting point is lower than the observed scatter for each size group with fixed beam intensity. The effect of the beam intensity is still under investigation. 


\section{WILEY-VCH}

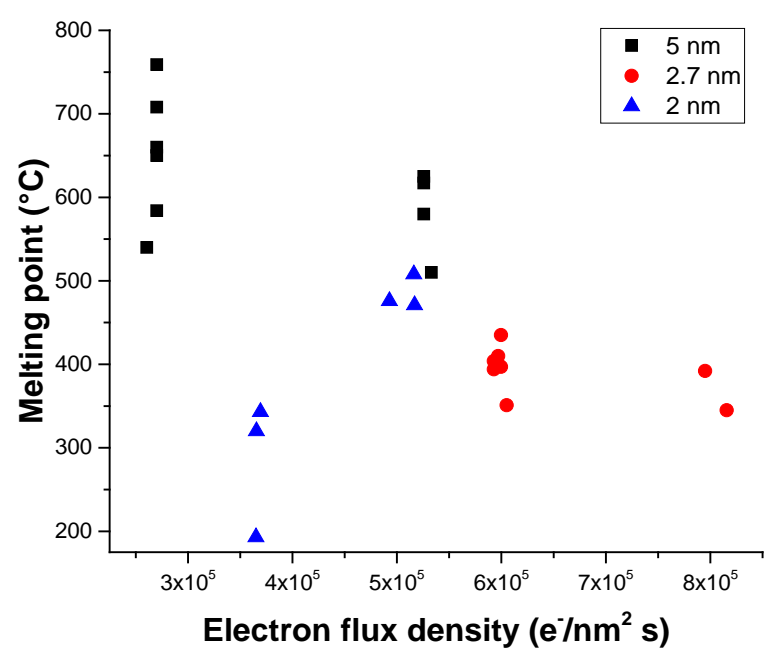

Figure 6: Experimentally determined melting temperatures at different electron beam intensities.

\section{Computational details}

Molecular dynamics (MD) simulations were performed using the Atomic Simulation Environment (ASE) [40]. The EMT potential [28] was implemented in the ASAP package, and the EAM potential for gold [29] was imported into ASAP from the Open Knowledgebase of Interatomic Models (OpenKIM) [41] model number MO_468407568810_002. Gold nanoparticles of different shapes were created: Wulff-constructions and round nanoparticles. The round particles were simply cut out of the bulk fcc structure. Surface energies of the fcc metals were taken from a DFT study reported in the literature [42] in order to create the Wulff-constructions. We considered (111), (100) and (110) surfaces. The particles were heated to the desired temperature using Langevin dynamics [43]. A friction parameter of $\gamma=$ $1.3 \cdot 10^{-18} \mathrm{~kg} / \mathrm{s}$ was used. Time steps to integrate the equations of motion were set to $5 \mathrm{fs}$ and the dynamics were run for 10 ns or more. To analyse the atomic arrangement of the particles, polyhedral template matching (PTM) was applied [31] with a cut-off for the scale-invariant Root-Mean-Square Deviation of RMSD $\mathrm{max}_{\max }=0.2$. 


\section{WILEY-VCH}

Least square regression has been used to fit the melting curves. The melting region has been fitted with a second order fit function $\left(\mathrm{f}(\mathrm{x})=\mathrm{ax} \mathrm{x}^{2}+\mathrm{bx}+\mathrm{c}\right)$. Cohesive energies are calculated as defined as $\mathrm{E}_{\mathrm{COH}}(\mathrm{NP})=\mathrm{E}(\mathrm{NP}) / \mathrm{N}-\mathrm{E}($ Atom), where $\mathrm{E} \mathrm{coH}(\mathrm{NP})$ is the cohesive energy of the nanoparticle, $\mathrm{E}(\mathrm{NP})$ is the total energy of the nanoparticle, $\mathrm{N}$ is the number of atoms in the nanoparticle and E(Atom) is the total energy of the respective metal atom in vacuum.

\section{Acknowledgements}

Financial support from the European Marie Curie Project CATSENSE (grant agreement number: 607417), the Villum Foundation V-SUSTAIN grant 9455 to the Villum Center for the Science of Sustainable Fuels and Chemicals and the Independent Research Fund Denmark project DynCat grant 12-133832 is gratefully acknowledged.

Received: ((will be filled in by the editorial staff))

Revised: ((will be filled in by the editorial staff)) Published online: ((will be filled in by the editorial staff)) 


\section{WILEY-VCH}

The depression of the melting point of nanoparticles compared to the bulk value is studied using transmission electron microscopy in combination with molecular dynamics simulations. The melting point is a crucial parameter for the optimized use of this category of materials. Understanding this fundamental property helps tailoring e.g. catalytic materials with superior performance.

Keyword: Melting point of Nanoparticles

P. Schlexer, A. B. Andersen, B. Sebok, I. Chorkendorff, J. Schiøtz and T. W. Hansen*

Size-dependence of the melting temperature of individual Au nanoparticles

ToC figure

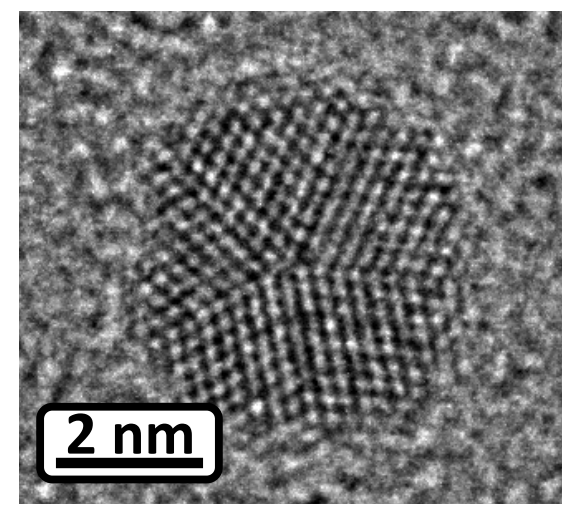




\section{WILEY-VCH}

Copyright WILEY-VCH Verlag GmbH \& Co. KGaA, 69469 Weinheim, Germany, 2013.

[1] M. C. Daniel and D. Astruc, Chem. Rev. 2004, 104, 293.

[2] K. K. Nanda, Pramana-J. Phys. 2009, 72, 617.

[3] A. Safaei, M. A. Shandiz, S. Sanjabi, and Z. H. Barber, J. Phys-Condens Mat. 2007, 19, 216216.

[4] J. H. Zhu, Q. S. Fu, Y. Q. Xue, and Z. X. Cui, J. Mater. Sci. 2016, 51, 4462.

[5] H. Li, P. D. Han, X. B. Zhang, and M. Li, Materials Chemistry and Physics 2013, 137, 1007.

[6] C. C. Yang and Y.-W. Mai, Materials Science and Engineering: R: Reports 2014, 79, 1.

[7] Z. H. Li and D. G. Truhlar, Chem. Sci. 2014, 5, 2605.

[8] G. Kaptay, J. Janczak-Rusch, G. Pigozzi, and L. P. H. Jeurgens, J. Mater. Eng. Perform. 2014, 23, 1600.

[9] F. Font, T. G. Myers, and S. L. Mitchell, Microfluid. Nanofluid. 2015, 18, 233.

[10] H. A. Alarifi, M. Atis, C. Ozdogan, A. Hu, M. Yavuz, and Y. Zhou, J. Phys. Chem. C 2013, 117, 12289.

[11] L. V. Sang, V. V. Hoang, and N. T. T. Hang, Eur. Phys. J. D 2013, 67, 8.

[12] I. L. Garzon and J. Jellinek, Zeitschrift Fur Physik D-Atoms Molecules and Clusters 1991, 20, 235.

[13] Q. Shu, Y. Yang, Y. T. Zhai, D. Y. Sun, H. J. Xiang, and X. G. Gong, Nanoscale 2012, 4, 6307.

[14] O. H. Nielsen, J. P. Sethna, P. Stoltze, K. W. Jacobsen, and J. K. Nørskov, Europhysics Letters 1994, 26, 51.

[15] B. Roldan Cuenya, M. Alcántara Ortigoza, L. K. Ono, F. Behafarid, S. Mostafa, J. R. Croy, K. Paredis, G. Shafai, T. S. Rahman, L. Li, Z. Zhang, and J. C. Yang, Phys. Rev. B 2011, 84, 245438.

[16] O. A. Yeshchenko, I. M. Dmitruk, A. A. Alexeenko, and A. M. Dmytruk, Phys. Rev. B 2007, 75, 085434.

[17] S. C. Hendy, Nanotechnology 2007, 18, 175703.

[18] P. Pawlow, Zeitschrift Fur Physikalische Chemie--Stochiometrie Und Verwandtschaftslehre 1909, 65, 545.

[19] E. Rie, Zeitschrift Fur Physikalische Chemie--Stochiometrie Und Verwandtschaftslehre 1923, 104, 354.

[20] H. Reiss and I. B. Wilson, Journal of Colloid Science 1948, 3, 551.

[21] P. Buffat and J.-P. Borel, Phys. Rev. A 1976, 13, 2287.

[22] S. Pratontep, S. J. Carroll, C. Xirouchaki, M. Streun, and R. E. Palmer, Rev. sci. Instrum. 2005, 76, 9.

[23] P. Hernandez-Fernandez, F. Masini, D. N. McCarthy, C. E. Strebel, D. Friebel, D. Deiana, P. Malacrida, A. Nierhoff, A. Bodin, A. M. Wise, J. H. Nielsen, T. W. Hansen, A. Nilsson, I. E. L. Stephens, and I. Chorkendorff, Nat. Chem. 2014, 6, 732.

[24] S. Ogawa and S. Ino, Journal of Vacuum Science and Technology 1969, 6, 527.

[25] K. Koga, T. Ikeshoji, and K. Sugawara, Phys Rev Lett 2004, 92, 115507.

[26] L. T. Roling and F. Abild-Pedersen, ChemCatChem 2018, 10, 1643.

[27] G. Ouyang, C. X. Wang, and G. W. Yang, Chem. Rev. 2009, 109, 4221.

[28] K. W. Jacobsen, P. Stoltze, and J. K. Norskov, Surf. Sci. 1996, 366, 394.

[29] X. W. Zhou, R. A. Johnson, and H. N. G. Wadley, Phys. Rev. B 2004, 69, 10.

[30] K. Michaelian and I. Santamaría-Holek, Europhysics Letters (EPL) 2007, 79, 43001. 


\section{WILEY-VCH}

[31] P. M. Larsen, S. Schmidt, and J. Schiøtz, Modelling and Simulation in Materials Science and Engineering 2016, 24, 055007.

[32] P. Vanysek, CRC Handbook of Chemistry and Physics, CRC Press, Boca Raton 1998.

[33] J. H. Shim, B. J. Lee, and Y. W. Cho, Surf. Sci. 2002, 512, 262.

[34] L. J. Lewis, P. Jensen, and J. L. Barrat, Phys. Rev. B 1997, 56, 2248.

[35] F. Guinea, J. H. Rose, J. R. Smith, and J. Ferrante, Appl. Phys. Lett. 1984, 44, 53.

[36] K. K. Nanda, S. N. Sahu, and S. N. Behera, Phys. Rev. A 2002, 66, 8.

[37] H. J. Duan, Y. Q. Xue, Z. X. Cui, Q. S. Fu, X. H. Chen, and R. Zhang, Part. Part. Syst. Charact. 2018, 35, 6.

[38] H. Haberland, M. Karrais, M. Mall, and Y. Thurner, Journal of Vacuum Science \& Technology a-Vacuum Surfaces and Films 1992, 10, 3266.

[39] B. von Issendorff and R. E. Palmer, Rev. sci. Instrum. 1999, 70, 4497.

[40] A. H. Larsen, J. J. Mortensen, J. Blomqvist, I. E. Castelli, R. Christensen, M. Dulak, J. Friis, M. N. Groves, B. Hammer, C. Hargus, E. D. Hermes, P. C. Jennings, P. B. Jensen, J. Kermode, J. R. Kitchin, E. L. Kolsbjerg, J. Kubal, K. Kaasbjerg, S. Lysgaard, J. B. Maronsson, T. Maxson, T. Olsen, L. Pastewka, A. Peterson, C. Rostgaard, J. Schiotz, O. Schutt, M. Strange, K. S. Thygesen, T. Vegge, L. Vilhelmsen, M. Walter, Z. H. Zeng, and K. W. Jacobsen, J. Phys-Condens Mat. 2017, $29,30$.

[41] E. B. Tadmor, R. S. Elliott, J. P. Sethna, R. E. Miller, and C. A. Becker, Jom 2011, 63, 17.

[42] N. E. Singh-Miller and N. Marzari, Phys. Rev. B 2009, 80, 235407.

[43] M. P. Allen and D. J. Tildesley, Computer Simulations of Liquids, Oxford University Press 1991. 